\title{
TRIVIALITY OF SIMPLE FIBER-PRESERVING ACTIONS OF TORI ON HILBERT-CUBE-FIBER BUNDLES
}

\author{
VO THANH LIEM
}

\begin{abstract}
Let $\sigma$ denote the standard based-free action of a torus $T$ on the Hilbert cube $Q$. It will be shown that every simple fiber-preserving action of $T$ on $Q \times B$, where $B$ is a finite-dimensional, compact metric absolute retract, is fiber-preserving equivalent to the diagonal action $\sigma \times$ id $_{B}$.
\end{abstract}

1. Introduction and definitions. Let $Q$ denote the Hilbert cube $I_{1} \times I_{2} \times \cdots$, and let $G$ be a topological group. An action $\alpha: G \times M \rightarrow M$ of $G$ on a topological space $M$ is said to be semifree if, for each $x \in M$, the isotropy group of $x$ is either $G$ of $\{e\}$. For a finite or compact Lie group $G$, it is known that the metric cone of $G, \operatorname{cone}(G)$, is a compact absolute retract (AR). The left translation on $G$ defines a natural semifree action of $G$ on $\operatorname{cone}(G)$ that induces a semifree action, the diagonal action of $G$ on $\prod_{n=1}^{\infty} \operatorname{cone}(G) \approx Q$ [We]. This action has a unique fixed point. Following [B-We], we call it the standard based-free action $\sigma$ of $G$ on $Q$ whose orbit space is denoted by $Q / \sigma$. An action $\lambda$ of $G$ on $Q \times B$ is said to be fiber-preserving (f.p.) if, for each $g \in G$, we have $p_{B} \circ \lambda(g, \cdot)=p_{B}(\cdot)$, where $p_{B}: Q \times B \rightarrow B$ is the projection. Two actions $\alpha$ and $\beta: G \times M \rightarrow M$ are said to be equivalent if there is a homeomorphism $f: M \rightarrow M$ such that

$$
f(\alpha(g, x))=\beta(g, f(x))
$$

for all $x \in M$ and $g \in G$. A f.p. action $\lambda$ on $Q \times B$ is said to be simple if the restriction of $\lambda$ to each fiber $Q \times\{b\}$ is equivalent to $\sigma$ and has fixed point set $\{(0, b)\}$.

In $[\mathbf{L}]$, the following result has been proved: "If $B$ is a finite-dimensional compact metric $A R$, then every simple f.p. action of a finite group $G$ on $Q \times B$ is equivalent to the diagonal action $\mathrm{\sigma} \times \mathrm{id}_{B}$ ".

The purpose of this paper is to establish a similar result for simple f.p. actions of tori on $Q \times B$, where $B$ is a finite-dimensional, compact metric $\mathrm{AR}$, as stated in the abstract or Theorem 6 below.

In this paper, we set $I=[0,1], Q_{0}=Q-\{0\}$. For basic notions and results in $Q$-manifold theory, we refer to $\left[\mathbf{C h}_{\mathbf{1}}\right]$. A map $p: X \rightarrow B$ is a Hurewicz fibration if $p$ has the homotopy covering extension property (HCEP) with respect to every

Received by the editors February 22, 1982 and, in revised form, June 28, 1982.

1980 Mathematics Subject Classification. Primary 57A20. 55F99: Secondary 58D05.

$\mathrm{Key}$ words and phrases. Hurewicz fibration. fiber bundle, $\alpha$-equivalence, semifree action, fiber-preserving map. reduced cone.

${ }^{1}$ Research partially supported by Grant No. 1045 of the University of Alabama. 
topological space. The following is a special case of the main result in $\left[\mathrm{Ch}_{2}\right]$ that we will use

Lemma 0. Let $p: X \rightarrow B$ be a Hurewicz fibration, where $B$ is a f.d. compact $A R$ and the fibers are $Q$-manifolds, then the composition $p \circ p_{X}: X \times[0,1) \rightarrow X \rightarrow B$ is $a$ locally trivial fiber bundle.

Given $\alpha$ : $G \times Q \times B \rightarrow Q \times B$ a simple f.p. action of $G$ over $B$, we will define $E$ to be its orbit space and $E_{0}$ its regular orbit space $E \backslash(\{0\} \times B)$ (we identify the fixed point set $\{0\} \times B$ with its image in the orbit space). Let $p: E \rightarrow B$ denote the natural map induced from the projection map $p_{B}: Q \times B \rightarrow B$. The fiberwise-reduced cone $C(E, p)$ of $E$ over $B$ is defined to be the quotient $(E \times I) / \mathscr{D}$, where $\mathscr{D}$ is the decomposition of $E \times I$ whose nondegenerate elements are all closed subsets of the form $\left(p^{-1}(b) \times\{1\}\right) \cup(b \times I)$ for $b \in B$. Let $d_{1}$ denote a metric on $C(E, p)$ which extends a metric, say $d_{1}$, in $E$. Note that, besides being endowed with the metric $d_{1}$, the open subset $E_{0} \times[0,1)$ of $C(E, p)$ also inherits the product metric $d_{2}$ of $d_{1} \mid E_{0}$ and the usual metric on $[0,1)$. Given a $\delta>0$, a $\delta$-subset of a metric space is a subset of diameter less than $\delta$.

ObSERVATIONS. Let $K$ be a compact subset of $E_{0}$ and let $\varepsilon$ be a positive number. Then, there is a $\delta>0$ such that every $\delta$-subset of $\left(E_{0} \times[0,1), d_{2}\right)$ intersecting $K$ is an $\varepsilon$-subset of $\left(E_{0} \times[0,1), d_{1}\right)$. Consequently, if $\rho: K \times I \rightarrow C(E, p)$ is a $\delta$-homotopy (i.e. $\left.\operatorname{diam}_{2} \rho(x \times I)<\delta\right)$ in $\left(E_{0} \times[0,1), d_{2}\right)$ with $\rho(x, 0)=x$ for all $x$ in $K$, then $\operatorname{diam}_{1} \rho(x \times I)<\varepsilon$ in $\left(E_{0} \times[0,1), d_{1}\right)$ for all $x$ in $K$. In particular, for the projection $p_{E}: C(E, p) \rightarrow E$ and a $\delta$-subset $A$ of $\left(E_{0} \times[0,1), d_{2}\right)$ intersecting $K$, then $\operatorname{diam}_{1} p_{E}(A)<\varepsilon$ in $\left(E, d_{1}\right)$. Similar properties also hold true when $d_{1}$ and $d_{2}$ are interchanged.

Given an open cover $\beta$ of a space $Y$, a homomotopy $H: X \times I \rightarrow Y$ is said to be $\beta$-homotopy if for each $x \in X$, the track $H(x \times I)$ is contained in a member of $\beta$; a map $f: X \rightarrow Y$ is said to be a $\beta$-equivalence if there is a map $g: Y \rightarrow X$ such that $f \circ g$ is $\beta$-homotopic to id $\mathrm{id}_{Y}$ and $g \circ f$ is $f^{-1}(\beta)$-homotopic to id ${ }_{X}$, where $f^{-1}(\beta)$ denotes the open cover $\left\{f^{-1}(U) \mid U \in \beta\right\}$ of $X$.

2. Results and details of proofs. In the proof of the following proposition, we refer to p. 266 of [Do] for the definition of section extension property (SEP), numerable open covering, etc.

Proposition 1. If $\lambda$ is a simple f.p. action of a finite group or a compact Lie group $G$ on $Q \times B$, then the natural map $p: E_{0} \rightarrow B$ is a Hurewicz fibration.

Proof. Since $E_{0}$ and $B$ are metric spaces, we only have to show that $p$ has the HCEP for the class of all metric spaces [Du, Corollary 2.3, p. 396]. Assume that $X$ is a metric space. Given a commutative diagram

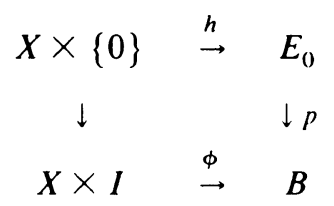


we will show that $\phi$ has a lifting $\Phi: X \times I \rightarrow E_{0}$ with $\Phi(x, 0)=h(x)$ for all $x$ in $X$. Equivalently, if we define

(1) $R=\left\{(x, w) \in X \times E_{0}^{\prime} \mid w(0)=h(x)\right.$ and $\left.p w(t)=\phi(x, t)\right\}$, and

(2) $q: R \rightarrow X$ by $q(x, w)=x$,

then we will show that there is a numerable open cover $\left\{V_{\lambda} \mid \lambda \in \Lambda\right\}$ of $X$ such that the restriction $q_{\lambda}=q \mid q^{-1}\left(V_{\lambda}\right): q^{-1}\left(V_{\lambda}\right) \rightarrow V_{\lambda}$ has SEP; hence. $q$ has a cross-section by Theorem 2.7 of [Do].

Let $\left\{U_{\lambda} \mid \lambda \in \Lambda\right\}$ be a covering of $E_{0}$ consisting of contractible open subsets. Let $V_{\lambda}=h^{-1}\left(U_{\lambda}\right), \lambda \in \Lambda$, then $\left\{V_{\lambda} \mid \lambda \in \Lambda\right\}$ is numerable since $X$ is a metric space. Let $\pi: Q_{0} \times B \rightarrow E_{0}$ denote the orbit map. Then, $\pi \mid \pi^{-1}\left(U_{\lambda}\right): \pi^{-1}\left(U_{\lambda}\right) \rightarrow U_{\lambda}$ is a trivial fiber bundle with fiber $G\left[\mathbf{B r}\right.$. Theorem 5.8, p. 88]; hence, there is a lifting $\tilde{h}_{\lambda}$ : $V_{\lambda} \times\{0\} \rightarrow \pi^{-1}\left(U_{\lambda}\right) \subset Q_{0} \times B$ of $h_{\lambda}=h \mid\left(V_{\lambda} \times\{0\}\right)$. Observe that the pair $\left(\phi_{\lambda}, \tilde{h}_{\lambda}\right)$, where $\phi_{\lambda}=\phi \mid\left(V_{\lambda} \times I\right)$, has the HCEP; consequently, so does the pair $\left(\phi_{\lambda}, h_{\lambda}\right)$. Therefore, the map $q_{\lambda}: q^{-1}\left(V_{\lambda}\right) \rightarrow V_{\lambda}$ has SEP by Lemma 4.5 of [Do]. So, the proof of the proposition is complete.

For the sake of simplicity, let $F_{h}$ denote $p^{-1}(b), X_{h}$ its reduced cone at $(0, b)$ and $F_{h .0}$ the regular orbit space $F_{h} \cap E_{0}$ over $b$ for each $b \in B$; let $Y_{h}$ denote $(Q / \sigma) \times\{b\}$. Let $d$ denote the product metric on $Q / \sigma \times B$. Given $b, c \in B$, let $\varepsilon_{h, c}: Y_{b} \rightarrow Y_{c}$ be the natural homeomorphism defined by $\varepsilon_{h, c}(x, b)=(x, c)$, then $d\left(\varepsilon_{h, c}(x, b),(x, b)\right)$ $=d_{B}(b, c)$ for each $(x, b) \in Y_{h}$.

From the above proposition and Lemma 0 , it follows that the composition $p \circ p_{E_{0}}: E_{0} \times[0,1) \rightarrow B$ is a trivial fiber bundle whose fiber is homeomorphic to $Q_{0} / \boldsymbol{\sigma}$ [B-We]. Therefore, $E_{0} \times[0,1)$ is f.p. homeomorphic to $\left(Q_{0} / \boldsymbol{\sigma}\right) \times B$ over $B$. Consequently, its fiberwise-one-point compactification $C(E, p)$ is f.p. homeomorphic to $(Q / \sigma) \times B$ over $B$. Let $h$ denote such a homeomorphism in the following Lemmas 2-5.

LEMMA 2. Given an $\varepsilon>0$, there is a $\delta>0$ such that if $d_{B}(b, c)<\delta$, then $d_{1}\left(h^{-1} \varepsilon_{h, c} h(x), x\right)<\varepsilon$ for all $x \in X_{b}$ and $d_{1}\left(h^{-1} \varepsilon_{c, b} h(z), z\right)<\varepsilon$ for all $z \in X_{c}$.

Proof. From the uniform continuity of $h^{-1}$, there is a $\delta>0(\delta<\varepsilon)$ such that $d\left(h^{-1}(y), h^{-1}\left(y^{\prime}\right)\right)<\varepsilon$ for every pair of $y, y^{\prime} \in(Q / \sigma) \times B$ with $d\left(y, y^{\prime}\right)<\delta$. Now, if $d_{B}(b, c)<\delta$, then for each $x \in X_{b}$ we have $d\left(\varepsilon_{h, c} h(x), h(x)\right)=d_{B}(b, c)<\delta$; hence, $d_{1}\left(h^{-1} \varepsilon_{b, c} h(x), x\right)=d_{1}\left(h^{-1} \varepsilon_{b, c} h(x), h^{-1} h(x)\right)<\varepsilon$ as we desired. The proof of the second inequality is the same.

LEMMA 3. Given an $\varepsilon>0$, there is a $\delta(0<\delta<\varepsilon)$ such that if $d_{B}(b, c)<\delta$ and if $A$ is a $\delta$-subset of $\left(X_{b}, d_{1}\right)$, then $h^{-1} \varepsilon_{h, c} h(A)$ is an $\varepsilon$-subset of $\left(C(E, p), d_{1}\right)$.

Proof. From Lemma 1 , choose a $\delta<\varepsilon / 3$ such that if $d_{B}(b, c)<\delta$, then $d_{1}\left(h^{-1} \varepsilon_{b . c} h(x), x\right)<\varepsilon / 3$ for each $x \in X_{b}$. Then, for $x, y \in X_{b}$ with $d_{1}(x, y)<\delta$, we have

$$
\begin{aligned}
d_{1}\left(h^{-1} \varepsilon_{b, c} h(x), h^{-1} \varepsilon_{b, c} h(y)\right) \leqslant & d_{1}\left(h^{-1} \varepsilon_{b, c} h(x), x\right)+d_{1}(x, y) \\
& +d_{1}\left(y, h^{-1} \varepsilon_{h, c} h(y)\right) \\
< & \varepsilon .
\end{aligned}
$$


Lemma 4. Fix $a$ point $b \in B$ and a compact subset $K$ of $F_{h}-\{b\}$. Given an $\varepsilon>0$, then there is a $\delta>0$ such that if $d_{B}(b, c)<\delta$, there are maps $f: F_{c} \rightarrow F_{b}$ and $g$ : $F_{h} \rightarrow F_{c}$ having the following properties:

(a) $d_{1}(f(x), x)<\varepsilon$ for all $x \in f^{-1}(K)$, and $d_{1}(g(y), y)<\varepsilon$ for all $y \in K$.

(b) $g \circ f \mid f^{-1}(K)$ is $\varepsilon$-homotopic to the inclusion $f^{-1}(K) \subset F_{c}$.

(c) $f \circ g \mid K$ is $\varepsilon$-homotopic to the inclusion $K \subset F_{h}$.

Sublemma. Let $M$ be a compact subset of $E_{0}$. Given an $\varepsilon>0$, there is an $\varepsilon_{1}<\varepsilon$ such that if $d_{B}(b, c)<\varepsilon_{1}$ and if $A$ is an $\varepsilon_{1}$-subset of $\left(X_{c}, d_{1}\right)$ intersecting $M$, then $p_{E} h^{-1} \varepsilon_{c . h} h(A)$ is an $\varepsilon$-subset of $F_{c}$.

Proof. Let $\eta$ be a positive number such that the closure $P$ of the $\eta$-neighborhood $N_{\eta}(M)$ of $M$ is a compact subset of $E_{0}$. Choose $\mu(0<\mu<\eta)$ such that $\operatorname{diam}_{1} p_{E}(R)$ $<\varepsilon$ for each $\mu$-subset $R$ of $\left(P \times[0, \mu], d_{1}\right)$. Then, choose $\varepsilon_{1}\left(0<\varepsilon_{1}<\mu / 2\right)$ such that if $d_{R}(b, c)<\varepsilon_{1}$, then

(*) $\operatorname{diam}\left(h^{-1} \varepsilon_{c, h} h(A)\right)<\mu / 2$ with respect to $d_{1}$ and $d_{2}$ for every $\varepsilon_{1}$-subset $A$ of $\left(X_{c}, d_{1}\right)$ intersecting $M$ (see Lemma 3 and Observations), and

(**) $d_{1}\left(h^{-1} \varepsilon_{c, h} h(x), x\right)<\mu / 2$ and $d_{2}\left(h^{-1} \varepsilon_{c, h} h(x), x\right)<\mu / 2$ for every $x \in F_{c} \cap$ $M$ (see Lemma 2 and Observations).

Now, if $A$ is an $\varepsilon_{1}$-subset of $\left(X_{c}, d_{1}\right)$ intersecting $M$, then $h^{-1} \varepsilon_{c, h} h(A)$ intersects $N_{\mu / 2}(M) \times[0, \mu / 2]$ by $(* *)$. Combining this fact and $(*)$, we observe that the set $R=h^{-1} \varepsilon_{c, h} h(A)$ is a $\mu$-subset of $\left(N_{\mu}(M) \times[0, \mu], d_{1}\right) \subset\left(P \times[0, \mu], d_{1}\right)$. Therefore, $\operatorname{diam}_{1}\left(p_{E} h^{-1} \varepsilon_{c, h} h(A)\right)<\varepsilon$ by the choice of $\mu$.

Let us return to the proof of Lemma 4 . Given an $\varepsilon>0$, we will choose inductively the positive numbers $\varepsilon>\varepsilon_{1}>\delta_{2}>\delta_{1}>\delta_{E}>\delta$ as follows: We can assume that $\varepsilon$ is so small that $N_{2 \varepsilon}(K) \cap N_{2 \varepsilon}(B)=\varnothing$, and let $M$ be the closure of $N_{2 \varepsilon}(K)$.

(1) Choose $\varepsilon_{1}$, from the Sublemma, such that if $H: Z \times I \rightarrow\left(E_{0} \times[0,1), d_{1}\right)$ is an $\varepsilon_{1}$-homotopy with $H(z \times I) \cap M \neq \varnothing$ and $H(z \times I) \subset X_{h}$ for all $z \in Z$, then $\left(p_{E} h^{-1} \varepsilon_{h, c} h\right) \circ H$ is an $\varepsilon$-homotopy in $\left(E, d_{1}\right)$ when $d_{B}(b, c)<\varepsilon_{1}$.

(2) Choose $\delta_{2}$ such that every $\delta_{2}$-homotopy $F: Z \times I \rightarrow\left(E_{0} \times[0,1), d_{2}\right)$ with $F(z \times I) \cap M \neq \varnothing$ for all $z \in Z$ is an $\varepsilon_{1}$-homotopy in $\left(E_{0} \times[0,1), d_{1}\right)$ (from Observations).

(3) Choose $\delta_{1}$ such that every $\delta_{1}$-subset of $\left(E_{0} \times[0,1), d_{1}\right)$ intersecting $M$ is a $\delta_{2}$-subset of $\left(E_{0} \times[0,1), d_{2}\right)$ (from Observations).

(4) Choose $\delta_{E}$ such that if $A$ is a $\delta_{E}$-subset of $\left(E_{0} \times[0,1), d_{1}\right)$ intersecting $M$, then $p_{E}(A)$ is a $\left(\delta_{1} / 2\right)$-subset of $\left(E, d_{1}\right)$.

(5) Choose $\delta$ such that if $d_{B}(b, c)<\delta$, then $d_{1}\left(h^{-1} \varepsilon_{c, b} h(x), x\right)<\delta_{E}$ for all $x \in X_{c}$ and $d_{1}\left(h^{-1} \varepsilon_{h, c} h(y), y\right)<\delta_{E}$ for all $y \in X_{h}$ (from Lemma 2).

With this choice of $\delta$, we now define $f=p_{E} h^{-1} \varepsilon_{c, h} h$ and $g=p_{E} h^{-1} \varepsilon_{h, c} h$ and show that $f$ and $g$ have all required properties if $d_{B}(b, c)<\delta$.

(a) For $x \in f^{-1}(K) \subset F_{c} \subset X_{c}$, then $d_{1}\left(h^{-1} \varepsilon_{c, b} h(x), x\right)<\delta_{E}$ by (5); hence, $d_{1}\left(p_{E} h^{-1} \varepsilon_{c, b} h(x), p_{E}(x)\right)<\delta_{1} / 2$ by (4); consequently, $d_{1}(f(x), x)<\delta_{1} / 2<\varepsilon$. Similarly, $d_{1}(g(y), y)<\varepsilon$ for $y \in K \subset F_{b}$. 
(b) Observe that if $x \in f^{-1}(K)$, then

$$
\begin{aligned}
d_{1}\left(p_{E} h^{-1} \varepsilon_{c, b} h(x), h^{-1} \varepsilon_{c, b} h(x)\right) & \leqslant d_{1}\left(p_{E} h^{-1} \varepsilon_{c, b} h(x), x\right)+d_{1}\left(x, h^{-1} \varepsilon_{c, b} h(x)\right) \\
& =d_{1}(f(x), x)+d_{1}\left(x, h^{-1} \varepsilon_{c, b} h(x)\right) \\
& <\delta_{1} / 2+\delta_{E}<\delta_{1}
\end{aligned}
$$

(from the proof of (a) and by (5)). Hence, $d_{2}\left(f(x), h^{-1} \varepsilon_{c, b} h(x)\right)<\delta_{2}$ by (3), since $f(x) \in M$. Therefore, $f$ is $\delta_{2}$-homotopic along $[0,1)$ to $h^{-1} \varepsilon_{c, b} h$ in $\left(F_{b, 0} \times[0,1), d_{2}\right)$ $\subset X_{b}$, say $H$. Consequently, $H$ is an $\varepsilon$-homotopy in $\left(X_{b}, d_{1}\right)$ by (2). Finally, $\left(p_{E} h^{-1} \varepsilon_{b, c} h\right) \circ H$ is an $\varepsilon$-homotopy by (1) from $g \circ f$ to

$$
p_{E} h^{-1} \varepsilon_{b, c} h h^{-1} \varepsilon_{c, b} h=p_{E} \mid f^{-1}(K)=\text { the inclusion } f^{-1}(K) \subset F_{c} \text {. }
$$

(c) The proof of (c) is the same as that of (b).

The proof of Lemma 4 is now complete.

LEMMA 5. Fix an element $b$ of $B$. Given an open cover $\beta$ of $F_{b}$ and an open subset $U$ of $F_{b}$ whose closure $K$ is contained in $F_{b, 0}$, then there is $a \delta>0$ such that, if $c \in B$ with $d_{B}(b, c)<\delta$, then there is a map $f: F_{c} \rightarrow F_{b}$ which is a $\beta$-equivalence over $U$.

Proof. Let $\lambda$ be a positive number such that every $\lambda$-subset of $F_{b}$ intersecting the compactum $K$ is contained in a member of $\beta$ meeting $K$. Let $\delta$ be a positive number from Lemma 4 corresponding to $\varepsilon=\lambda / 4$; and let $f$ and $g$ be two maps satisfying (a), (b) and (c) in Lemma 4.

Now, it is clear that $f \circ g \mid K$ is $\beta$-homotopic to the inclusion $K \subset F_{b .0}$ by (c). Moreover, there is a $(\lambda / 4)$-homotopy $H$ from $g \circ f \mid f^{-1}(K)$ to the inclusion $f^{-1}(K)$

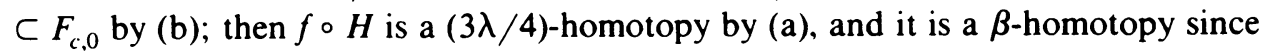
each tract intersects $K$. Therefore, $f$ is a $\beta$-equivalence over $U$ as we desired.

THEOREM 6. Let $B$ be a finite-dimensional compact metric $A R$. If $\lambda$ is a simple f.p. action of the torus $T^{n}$ on $Q \times B$, then (1) the map $p: E \rightarrow B$ is a locally trivial fiber bundle, and (2) $\lambda$ is $f$.p. equivalent to the diagonal action $\sigma \times \mathrm{id}_{K}$.

Proof. The proof will be similar to that of Theorem 3.2 in [L].

(1) Since the homeomorphism group $\operatorname{Homeo}(Q / \sigma)$ is locally contractible $[\mathbf{L}$, Theorem 2.2], we will only have to show that the map $p$ is completely regular; i.e. given an element $b \in B$ and an $\varepsilon>0$, we will find a $\delta>0$ such that, for each $c \in B$ with $d_{B}(b, c)<\delta$, there is a homeomorphism $\phi: F_{c} \rightarrow F_{b}$ such that $d_{1}(\phi(x), x)<\varepsilon$ for each $x \in F_{c}$.

Following [B-We], we will use the following notation in this proof. Let $L_{n}$ denote the orbit space $(T * T * \cdots * T) / \alpha$ of the induced diagonal action $\alpha$ (from the left translation of $T$ on itself) on the join of $n$ copies of $T$. Then, $L_{n}$ is naturally embedded in $L_{n+1}$, and let $M_{n}$ denote the mapping cylinder

$$
\operatorname{Map}\left(L_{1} \rightarrow L_{2} \rightarrow \cdots \rightarrow L_{n}\right)
$$

Define $M_{\infty}=\cup_{1}^{\infty} M_{n}$. It is proved in [B-We] that $F_{b}$ is homeomorphic to the one-point compactification of $M_{\infty} \times Q$. 
Given an $\varepsilon>0$, there is an integer $m$ such that $F_{b}-\left(M_{m} \times Q\right)$ is contained in $N_{\varepsilon / 2}(0, b)$, the $(\varepsilon / 2)$-neighborhood of $(0, b)$. Let $\varepsilon^{\prime}\left(0<\varepsilon^{\prime}<\varepsilon / 2\right)$ be chosen such that $M_{m+2} \times Q$ misses $N_{\varepsilon^{\prime}}(0, b)$.

Let $\beta$ be an open cover of $F_{b}$ as in Theorem 3.6 in [F] such that every $\beta$-equivalence $f: N \rightarrow F_{h .0}\left(N\right.$ is a $Q$-manifold) over the interior of $M_{m+2} \times Q$ is $\left(\varepsilon^{\prime} / 2\right)$-homotopic to a map $\bar{f}: N \rightarrow F_{b, 0}$ having the following properties:

(a) $\bar{f} \mid \bar{f}^{-1}\left(M_{m+1} \times Q\right)$ is an open embedding, and

(b) $\bar{f}(x)=f(x)$ for all $x \in N-f^{-1}\left(M_{m+2} \times Q\right)$.

Now, choose $\delta\left(0<\delta<\varepsilon^{\prime} / 2\right)$ as in Lemma 5 corresponding to the open cover $\beta$ and $U=$ int $M_{m+2} \times Q$. Let $b$ and $c$ be in $B$ with $d_{B}(b, c)<\delta$, and let $f: F_{c} \rightarrow F_{b}$ be a $\beta$-equivalence given by Lemma 5 , and let $\bar{f}$ be as in the above paragraph. Observe that $\bar{f}^{-1}\left(L_{m+1 / 2} \times Q\right)$ is bicollared in $F_{c, 0}$ and it is contained in $N_{\varepsilon}(0, c)$. Then, as in the proof of Theorem 3.2 in [L], since $F_{c, 0}$ and $F_{b, 0}$ are of the homotopy type of the Eilenberg-Mac Lane space $K\left(Z^{n}, 2\right)$, we can extend $\bar{f} \mid \bar{f}^{-1}\left(M_{m+1 / 2} \times Q\right)$ to a homeomorphism $\phi: F_{c, 0} \rightarrow F_{b, 0}$, and finally from $F_{c}$ onto $F_{b}$. Finally, it can be verified that $d(\phi(x), x)<\varepsilon$ for all $x \in F_{c}$. Therefore, the proof of (1) is complete.

(2) By (1), we now identify $E_{0}$ with $\left(Q_{0} / \sigma\right) \times B$. Since the principal $T$-bundle $p_{1}$ : $Q_{0} \times B \rightarrow E_{0}=\left(Q_{0} / \sigma\right) \times B$ is universal and $B$ is contractible the action is classified by the projection map $E_{0}=Q_{0} / \sigma \times B \rightarrow Q_{0} / \sigma \times\left\{b_{0}\right\}$ for any $b_{0} \in B$ and is therefore equivalent to $\sigma \times \mathrm{id}_{B}$ by a fiber-preserving, equivariant homeomorphism $g: Q_{0} \times B \rightarrow Q_{0} \times B$, which must extend to a homeomorphism of $Q \times B$.

\section{REFERENCES}

[B-We] I. Berstein and J. E. West, Based-free compact Lie group actions on Hilhert cube, Proc. Sympos. Pure Math., vol. 32, Part 1, Amer. Math. Soc., Providence, R. I., pp. 373-391.

[Br] G. E. Bredon, Introduction to compact transformation groups, Pure and Applied Math. Series, no. 46. Academic Press, New York, 1972.

$\left[\mathrm{Ch}_{1}\right]$ T. A. Chapman, Lectures on Hilhert cube manifolds, CBMS Conf. Ser. in Math., no. 28. Amer. Math. Soc., Providence, R. I., 1976.

$\left[\mathrm{Ch}_{2}\right]$ _. Proper fibrations with Hilhert cube manifold fibers, Topology Appl. 12 (1981), 19-33.

[Do] A. Dold, Partition of unity in the theory of fibrations, Ann. of Math. (2) 78 (1963), 223-255.

[Du] J. Dugundji, Topologi, Allyn \& Bacon, Boston, Mass., 1973.

[F] S. Ferry. The homeomorphism group of a compact Hilhert cube manifold is an ANR. Ann. of Math. (2) 106 (1977), 101-119.

[L] V. T. Liem, Some results on semi-free actions of finite groups on Hilbert cube manifolds, Topology Appl. 12 (1981), 147-159.

[We] J. E. West, Infinite products which are Hilhert cubes, Trans. Amer. Math. Soc. 150 (1970), 1-25.

Department of Mathematics, University of Alabama, University, Alabama 35486 